\title{
Anti-inflammatory actions of gabapentin and pregabalin on the substance P-induced mitogen-activated protein kinase activation in U373 MG human glioblastoma astrocytoma cells
}

\author{
KEISUKE YAMAGUCHI $^{1}$, SEIICHIRO KUMAKURA ${ }^{1}$, AKIMASA SOMEYA ${ }^{2}$, \\ MASAKO ISEKI $^{1}$, EIICHI INADA ${ }^{1}$ and ISAO NAGAOKA ${ }^{2}$ \\ Departments of ${ }^{1}$ Anesthesiology and Pain Medicine and ${ }^{2}$ Host Defense and Biochemical Research, \\ Juntendo University Graduate School of Medicine, Tokyo 113-8421, Japan
}

Received February 16, 2017; Accepted August 3, 2017

DOI: $10.3892 / \mathrm{mmr} .2017 .7368$

\begin{abstract}
Gabapentin (GBP) and pregabalin (PGB) exert antinociceptive effects on chronic nociceptive responses with neuropathic or inflammatory conditions. Furthermore, it is considered that GBP and PGB exhibit anti-inflammatory effects by modulating the substance $\mathrm{P}$ (SP)-mediated neurokinin-1 receptor (NK1R; a SP receptor) response. Thus, in the present study, the effects of GBP and PGB on SP-induced activation were investigated in the human glioblastoma astrocytoma U373 MG cell line, which expresses high levels of functional high-affinity NK1R, and produces interleukin (IL)-6 and IL-8 in response to SP. The results indicated that GBP and PGB suppressed the SP-induced production of IL-6, and IL-8 in U373 MG cells. Furthermore, GBP and PGB inhibited the SP-induced phosphorylation of p38 mitogen-activated protein kinase (MAPK) and nuclear factor $(\mathrm{NF})-\kappa \mathrm{B}$, and the nuclear translocation of NF- $\kappa \mathrm{B}$ in U373 MG cells. Together, these observations suggest that GBP and PGB likely prevent SP-induced IL-6 and IL-8 production in U373 MG cells via the inhibition of signaling molecules, including p38 MAPK and $N F-\kappa B$, thereby exhibiting antineuroinflammatory effects.
\end{abstract}

\section{Introduction}

A neuropeptide substance $\mathrm{P}$ (SP) is an important mediator of neurogenic inflammation in the central and peripheral nervous systems. SP is implicated in pain, and also plays an important role in tumor cell proliferation, anti-apoptotic effects on tumor cells, angiogenesis, tumor cell invasion and metastasis (1-3). SP

Correspondence to: Professor Isao Nagaoka, Department of Host Defense and Biochemical Research, Juntendo University Graduate School of Medicine, 2-1-1 Hongo, Bunkyo-ku, Tokyo 113-8421, Japan

E-mail: nagaokai@juntendo.ac.jp

Key words: gabapentin, pregabalin, substance P, p38 MAPK, NF- $\kappa$ B, NK-1 receptor, IL-6, IL-8, U373 MG cell, anti-inflammatory action is released from primary afferent nociceptors and sympathetic postganglionic neurons, and activates neighboring receptors, thereby triggering spreading activation $(4,5)$. Moreover, SP has been shown to induce the expression of proinflammatory cytokines and chemokines, such as interleukin (IL)-6 and IL-8 $(6,7)$, which are involved in the pathogenesis of several human brain disorders (such as multiple sclerosis, dementia complex, and Alzheimer's disease) (8), although it is currently a matter of debate whether SP really plays a pathogenic role in these disorder. Previous report has shown that the activation of a SP receptor [neurokinin-1 receptor (NK1R)] elicits signals affecting inflammatory cytokine gene expression (7). In addition, it has been reported that SP potently triggers the activation of nuclear factor $(\mathrm{NF})-\kappa \mathrm{B}$, an important transcriptional activator, which regulates the production of various cytokines and other proinflammatory mediators (7).

Mitogen-activated protein kinases (MAPK), a family of protein Ser/Thr kinases, consist of at least three major subfamilies: i) the p42/44 MAPKs, which are also called extracellular signal regulated kinases (ERK-1 and ERK-2); ii) the c-Jun NH2-terminal kinase/stress-activated protein kinases (JNK/SAPK) including p46 JNK1 and p54 JNK2; and iii) the p38 MAPK subfamily. MAPKs are activated under stress conditions in response to a variety of extracellular stimuli, including oxidative stress. Among these MAPKs, phosphorylation of $\mathrm{p} 38 \mathrm{MAPK}$ is induced in dorsal horn of the spinal cord and dorsal root ganglia following peripheral nerve injury or inflammation (9-11).

Gabapentin (GBP) and pregabalin (PGB) are structural analogues of $\gamma$-amino butyric acid (GABA) with lipophilic characteristics, and were developed as potential anticonvulsants (12). However, many studies have shown that these substances do not act as GABA agonists and indeed have little demonstrable effect on any aspect of GABA transmission $(13,14)$. Furthermore, a recent study has clarified the current understanding of gabapentinoid pharmacology that GBP and PGB do not inhibit any conventional subtype of voltage-gated calcium channel (VGCC), but rather selectively block calcium channels that contain the $\alpha 2 \delta-1$ subunit, with pharmacodynamics and cellular-specificities, depending on the structural and biochemical states of the $\alpha 2 \delta-1$ protein (15). 
It is believed that blockade of VGCC containing the $\alpha 2 \delta-1$ subunit is the predominant pharmacological mechanism of both GBP and PGB (16). PGB binds potently to the $\alpha 2 \delta-1$ subunit and modulates calcium influx at nerve terminals, thereby, reducing the release of several neurotransmitters, including glutamate, noradrenaline, serotonin and SP (17-19). Interestingly, GBP could be a therapeutic agent, when given systemically, for treatment of neuropathic or postsurgical pain (20-24), and also reduces the experimental pain in humans after sensitization of the skin with capsaicin and heat (25). Furthermore, GBP and its derivative PGB reduce nociceptive behaviors of animal models with neuropathic pain or inflammation such as nerve ligation, injection of immune antigens, herpes infection, arthritis, diabetes, postoperative pain and thermal injury (26-30). In contrast, neither GBP nor PGB alters acute nociceptive responses $(31,32)$. Thus, it is believed that the antinociceptive action of these substances depends on the chronic nociceptive responses with neuropathic or inflammatory conditions.

It has been demonstrated that the release of peptidergic neurotransmitters (including SP) from sensory neurons is increased during inflammation or in neuropathic pain models (33). In addition, SP is axonally transported to peripheral nerve endings, where it is released in response to traumatic stimuli and induces various biological effects (33), Importantly, GBP and PGB modulate the release of SP under the conditions corresponding to significant inflammation-induced sensitization of the spinal cord, which involves the action of SP on NK1 (SP) receptor (NK1R) (34). Based on the findings, we hypothesized that GBP and PGB may exert antiinflammatory action by modulating the SP-mediated NK1R response. In this study, therefore, we investigated the antiinflammatory effects of GBP and PGB on SP-induced activation of a human glioblastoma astrocytoma cell line U373 MG cell, which expresses the high levels of NK1R.

\section{Materials and methods}

Materials. U373 MG cell line (Uppsala; ECACC08061901) was purchased from European Collection of Cell Cultures (ECACC; Salisbury, UK). SP was obtained from Sigma-Aldrich Co., LLC., (St. Louis, MO, USA); GBP from Tokyo Chemical Industry Co., Ltd. (Tokyo, Japan); PGB from Toronto Research Chemicals (Toronto, ON, Canada); Minimum essential medium (MEM), non-essential amino acids (NEAA), sodium pyruvate and fetal bovine serum (FBS) from Gibco BRL Life Technologies (Grand Island, NY, USA). Phosphate-buffered saline (PBS), RIPA buffer containing protease inhibitor cocktail, sample buffer solution containing reducing reagent $(6 X)$ for SDS-PAGE, running buffer solution (10X) for SDS-PAGE, Blocking One, WB Stripping Solution Strong, and Protein Ladder One Multi-color (Broad Range) for SDS-PAGE from Nacalai Tesque, Inc., (Kyoto, Japan). BCA protein assay reagent kit and enhanced chemiluminescence reagent, SuperSignal West Dura and NE-PER nuclear and cytoplasmic extraction reagents from Thermo Fisher Scientific, Inc. (Waltham, MA, USA). Mini-PROTEAN ${ }^{\circledR}$ TGX $^{\mathrm{TM}}$ Precast Gel and Trans-Blot ${ }^{\circledR}$ Turbo $^{\text {TM }}$ Mini PVDF Transfer Packs from Bio-Rad Laboratories, Inc., (Hercules, CA, USA).
Antibodies. Anti-phospho-ERK1/2 MAPK (Thr202/Tyr204) rabbit antibody (no. 9101), anti-ERK1/2 MAPK rabbit antibody (no. 9102), anti-phospho-NF-кB p65 (Ser536) rabbit monoclonal antibody (mAb; no. 3033), anti-NF- $\mathrm{B}$ p65 rabbit $\mathrm{mAb}$ (no. 8242), and histone H3 (D1H2) XP rabbit mAb (no. 4499) from Cell Signaling Technology, Inc. (Danvers, MA, USA), anti-phospho p38 MAPK rabbit antibody (Thr180/Tyr182; V121A) from Promega Corporation (Madison, WI, USA), anti-p38 MAPK (p38/SAPK2 $\alpha$ ) mouse mAb (no. 612168) from BD Biosciences (San Jose, CA, USA). Horseradish peroxidase (HRP)-conjugated goat anti-rabbit IgG (AP132P), HRP-conjugated goat anti-mouse IgG/IgM (AP308P) from Chemicon International (Temecula, CA, USA).

Cell culture. U373 MG cells were cultured in MEM supplemented with $1 \%(\mathrm{v} / \mathrm{v})$ penicillin/streptomycin, and NEAA, $1 \mathrm{mM}$ sodium pyruvate and $10 \%$ heat-inactivated FBS. The cells were maintained at $37^{\circ} \mathrm{C}$ in a $5 \% \mathrm{CO}_{2}$ humidified atmosphere.

Preparation of whole cell lysate and western blot analysis. U373 MG cells were plated into 12-well tissue culture plates at a density of $1 \times 10^{5}$ cells/well and incubated in MEM with $10 \%$ FBS for $12 \mathrm{~h}$, followed by incubation in MEM with $0.5 \%$ FBS for $12 \mathrm{~h}$ at $37^{\circ} \mathrm{C}$. Subsequently, the cells were incubated with GBP or PGB (1 mM) for $60 \mathrm{~min}$, and then stimulated with SP (100 $\mathrm{nM})$ for 10 or $15 \mathrm{~min}$. Thereafter, the cells were washed three times with ice-cold PBS and lysed in $0.1 \mathrm{ml}$ of RIPA buffer $(50 \mathrm{mmol} / 1$ Tris- $\mathrm{HCl} \mathrm{pH} \mathrm{7.6,} 150 \mathrm{mmol} / 1$ $\mathrm{NaCl}, 1 \%$ Nonidet $\mathrm{P} 40,0.5 \%$ sodium deoxycholate, $0.1 \%$ SDS and Protease Inhibitor Cocktail). Protein concentrations of cell lysates were measured with BCA protein assay reagent (Thermo Scientific). The lysates were mixed with SDS-polyacrylamide gel electrophoresis (PAGE) sample buffer (62.5 mM Tris-HCl, pH 6.8, 2\% SDS, 10\% glycerol, $0.05 \%$ bromphenol blue, and 5\% 2-mercaptoethanol), and applied to SDS-PAGE in $10 \%$ gels (Mini-PROTEAN ${ }^{\circledR}$ TGX $^{\text {TM }}$ Precast Gel; 10-20 $\mu \mathrm{g}$ protein/lane). Thereafter, separated proteins were electroblotted onto polyvinylidine fluoride membranes (Trans-Blot ${ }^{\circledR}$ Turbo $^{\mathrm{TM}}$ Mini PVDF Transfer Packs). After incubation with Blocking One (Nacalai Tesque, Inc.), blots were proved with a 1,000-fold dilution of rabbit anti-phospho p38 MAPK antibody, anti-phospho ERK1/2 antibody or anti-phospho $\mathrm{NF}-\kappa \mathrm{B}$ antibody, and further proved with a 10,000-fold dilution of HRP-conjugated goat anti-rabbit IgG. Signals were detected with SuperSignal West Dura Chemiluminescent Substrate (Thermo Fisher Scientific, Inc.), and quantified using LAS-3000 luminescent image analyzer (Fujifilm, Tokyo, Japan) and MultiGauge software (Fujifilm). Thereafter, the antibody was stripped using WB Stripping Solution Strong (Nacalai Tesque, Inc.) at room temperature for $15 \mathrm{~min}$. Blots were proved with a 1,000-fold dilution of mouse anti-p38 MAPK antibody, rabbit anti-ERK1/2 antibody or anti-NF- $\mathrm{B}$ antibody, and further proved with a 10,000-fold dilution of HRP-conjugated goat anti-rabbit IgG or HRP-conjugated goat anti-mouse IgG/IgM. Signals were detected and analyzed, as described above.

Preparation of nuclear extract and western blot analysis. U373 MG cells were plated into 12-well tissue culture plates at 
A

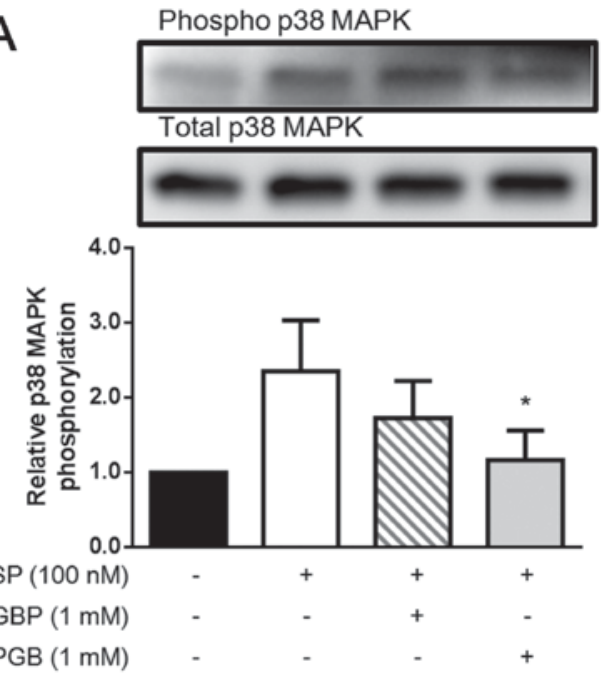

B
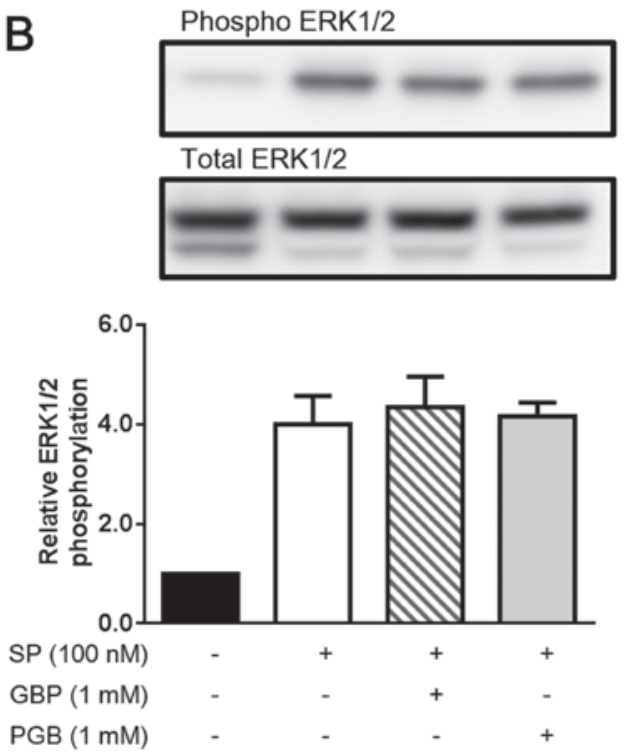

Figure 1. Evaluation of the effects of gabapentin (GBP) and pregabalin (PGB) on substance P (SP)-induced phosporylation of p38 MAPK and ERK1/2. U373 MG cells were plated into 12-well tissue culture plates at a density of $2 \times 10^{5}$ cells/well and incubated in MEM with $10 \%$ FBS for $12 \mathrm{~h}$, followed by incubation in MEM with $0.5 \%$ FBS for $12 \mathrm{~h}$ at $37^{\circ} \mathrm{C}$. Subsequently, the cells were incubated with or without GBP $(1 \mathrm{mM})$ or PGB $(1 \mathrm{mM})$ for $60 \mathrm{~min}$, and then incubated with or without SP $(100 \mathrm{nM})$ for $10 \mathrm{~min}$. (A) The phosphorylation of $\mathrm{p} 38$ MAPK was detected by probing with anti-phosphor p38 MAPK antibody and HRP-conjugated goat anti-rabbit IgG. In order to confirm that equal amount of proteins were analyzed in each samples, the blots were stripped, and total p38 MAPK were detected by reprobing with anti-p38 MAPK antibody and HRP-conjugated goat anti-mouse IgG/ IgM. (B) The phosphorylation of ERK1/2 were detected by probing with anti-phospho-ERK1/2 (Thr202/Tyr204) antibody and HRP-conjugated goat anti-rabbit IgG. Total ERK1/2 were detected by reprobing with anti-ERK1/2 antibody and HRP-conjugated goat anti-rabbit IgG. A representative image is shown. Data are the mean \pm standard deviation of 3 separate experiments, and expressed as relative to the cells incubated without SP, GBP, and PGB. Data are compared between the SP-stimulated cells incubated without and with GBP or PGB. "P $<0.05$.

a density of $2 \times 10^{5}$ cells/well and incubated in MEM with $10 \%$ FBS for $12 \mathrm{~h}$, followed by incubation in MEM with $0.5 \% \mathrm{FBS}$ for $12 \mathrm{~h}$ at $37^{\circ} \mathrm{C}$. Subsequently, the cells were incubated with GBP or PGB $(1.0 \mathrm{mM})$ for $60 \mathrm{~min}$, and then stimulated with
$\mathrm{SP}(100 \mathrm{nM})$ for $15 \mathrm{~min}$. Thereafter, the cells were washed three times with ice-cold PBS. Cells were detached by trypsin treatment, and washed in ice-cold PBS containing phosphatase inhibitors, and then centrifuged at $300 \mathrm{~g}$ for $5 \mathrm{~min}$. Nuclear fractions were prepared using NE-PER nuclear and cytoplasmic extraction reagents by suspending the cell pellet in a hypotonic buffer, and centrifugation at $14,000 \mathrm{x}$ g for $30 \mathrm{~min}$. After collection of the supernatant (cytoplasmic fraction), the pellet (nuclear fraction) was lysed and solubilized in lysis buffer containing proteasome inhibitors. Protein concentrations were determined using with BCA protein assay reagent. The nuclear extract was mixed with SDS-PAGE sample buffer), and applied to SDS-PAGE in $10 \%$ gels, followed by western blot analysis using a 1,000-fold dilution of rabbit anti-NF- $\kappa$ B antibody and a 10,000-fold dilution of HRP-conjugated goat anti-rabbit IgG, and a 1,000-fold dilution of rabbit histone H3 and a 10,000-fold dilution of HRP-conjugated goat anti-rabbit $\mathrm{IgG}$, as described in the above section.

Quantification of IL-6 and IL-8. U373 MG cells were plated into 12 -well tissue culture plates at a density of $1 \times 10^{5}$ cells/well and incubated in MEM with $10 \%$ FBS for $12 \mathrm{~h}$, followed by incubation in MEM with $0.5 \%$ FBS for $12 \mathrm{~h}$ at $37^{\circ} \mathrm{C}$. Then, the cells were incubated with GBP or PGB $(1 \mathrm{mM})$ for $60 \mathrm{~min}$, and stimulated with $\mathrm{SP}(100 \mathrm{nM})$ for $24 \mathrm{~h}$. Culture media were recovered, centrifuged for $10 \mathrm{~min}$ at $12,000 \mathrm{x} \mathrm{g}$, and the levels of IL-6 in the supernatants were measured by a sandwich enzyme-linked immunosorbent assay (ELISA) kit, according to the manufacturer's instructions (eBioscience, San Diego, CA, USA). Moreover, the levels of IL- 8 in the media were measured by ELISA kit, according to the manufacturer's instructions (R\&D systems, Inc., Minneapolis, MN, USA).

Statistical analysis. Data are expressed as mean \pm standard deviation, and analyzed for significant difference by a one-way ANOVA with multiple comparison test using GraphPad Prism version 6.0 for Windows (GraphPad Software, San Diego, CA, USA). $\mathrm{P}<0.05$ was considered to indicate a statistically significant difference.

\section{Results}

Suppression of SP-induced activation of p38 MAPK and $E R K 1 / 2$ by GBP and PGB. First, we evaluated the effect of GBP and PGB on the phosphorylation of p38 MAPK. As shown in Fig. 1A, SP stimulation (100 nM) clearly induced the phosphorylation of p38 MAPK in U373 MG cells. Interestingly, GBP (1 mM) and PGB (1 mM) substantially suppressed the SP-induced phosphorylation of p38 MAPK, although only the suppression by PGB was statistically significant $(\mathrm{P}<0.05)$.

Next, we evaluated the effect of GBP and PGB on the phosphorylation of ERK1/2. As shown in Fig. 1B, SP stimulation (100 $\mathrm{nM}$ ) markedly induced the phosphorylation of ERK1/2 in U373 MG cells; however, GBP (1 mM) and PGB (1 mM) did not essentially suppress the SP-induced phosphorylation of ERK1/2.

Suppression of SP-induced activation of $N F-\kappa B$ and nuclear translocation of $p 65$ by GBP and PGB. Furthermore, we 
evaluated the effect of GBP and PGB on the phosphorylation of NF- $\kappa$ B. SP stimulation (100 nM) substantially induced the phosphorylation of NF- $\kappa \mathrm{B}$ in U373 MG cells. Importantly, GBP and PGB significantly abolished the SP-induced phosphorylation of NF- $\kappa \mathrm{B}$ (Fig. 2A; $\mathrm{P}<0.05$ ).

To further characterize the effect of GBP and PGB on the SP-induced activation of NF- $\mathrm{KB}$, we evaluated the nuclear translocation of NF-kB. Thus, U373 cells were pretreated with GBP $(1 \mathrm{mM})$ and PGB $(1 \mathrm{mM})$ followed by stimulation with SP $(100 \mathrm{nM})$, and the nuclear levels of p65 were analyzed. Nuclear p65 level was increased after SP stimulation compared with unstimulated cells; however, SP-induced p65 level was significantly reduced by GBP and PGB (Fig. 2B; P<0.05).

Suppression of SP-induced IL- 6 and IL-8 production by GBP and $P G B$. Finally, we evaluated the effect of GBP and PGB on the IL- 6 and IL- 8 production by U373 MG cells. As shown in Fig. 3, both IL-6 and IL-8 were produced by resting U373 MG cells without SP. Importantly, the production of IL-6 and IL-8 was substantially increased by SP stimulation (100 nM). Noticeably, GBP and PGB $(1 \mathrm{mM})$ significantly suppressed both the IL- 6 and IL-8 production $(\mathrm{P}<0.05)$.

\section{Discussion}

To our knowledge, this is the first study to demonstrate the effects of GBP and PGB on the SP-induced inflammatory responses in glioblastoma astrocytoma U373 MG cells. U373 MG glioblastoma astrocytoma cells express a functional high-affinity NK1R (a SP receptor) (35) and are able to produce IL-6 and IL-8 in response to SP (6). In this study, we revealed that GBP and PGB suppressed the SP-induced production of IL-6 and IL-8 in U373 MG cells. Furthermore, GBP and PGB inhibited the SP-induced phosphorylation of p38 MAPK and NF- $\kappa B$, and nuclear translocation of NF- $\kappa B$ in U373 MG cells. Thus, GBP and PGB likely prevent the SP-induced IL- 6 and IL-8 production in U373 MG cells by inhibiting signaling molecules including p38 MAPK and NF- $\mathrm{BB}$, thereby exhibiting anti-inflammatory action.

According to pharmacokinetic study (36,37), the maximum plasma concentration of GBP after administration of a single $400 \mathrm{mg}$ GBP tablet to 12 volunteers was $3.33 \pm 1.19 \mu \mathrm{g} / \mathrm{ml}$ (approximately $20 \mu \mathrm{M}$ ). Moreover, it has been shown that $25 \mu \mathrm{M}$ GBP and PGB reduced the SP-induced NF- $\kappa B$ activation in glioblastoma cell line in vitro (38). In our preliminary experiments, we observed that $100 \mu \mathrm{M}$ GBP weakly but $1 \mathrm{mM}$ GBP clearly suppressed the SP-induced phosphorylation of p38 MAPK and NF- $\kappa B$, and production of IL-6 and IL-8. Thus, we examined the effect of GBP and PGB at a concentration of $1 \mathrm{mM}$ on the SP-induced phosphorylation of signaling molecules, and production of cytokines.

The concentration of GBP used in this study $(1 \mathrm{mM})$ was 50 -fold higher than that of the maximum plasma concentration; however, we observed that GBP at $100 \mu \mathrm{M}$ weakly suppressed the SP-induced phosphorylation of p38 MAPK and NF- $\kappa B$, and production of IL-6 and IL-8 (data not shown). Thus, we speculate that GBP could exert the anti-inflammatory action in vivo, based on our findings.

GBP and PGB are anticonvulsants originally developed as spasmolytic agents for the management of generalized or partial
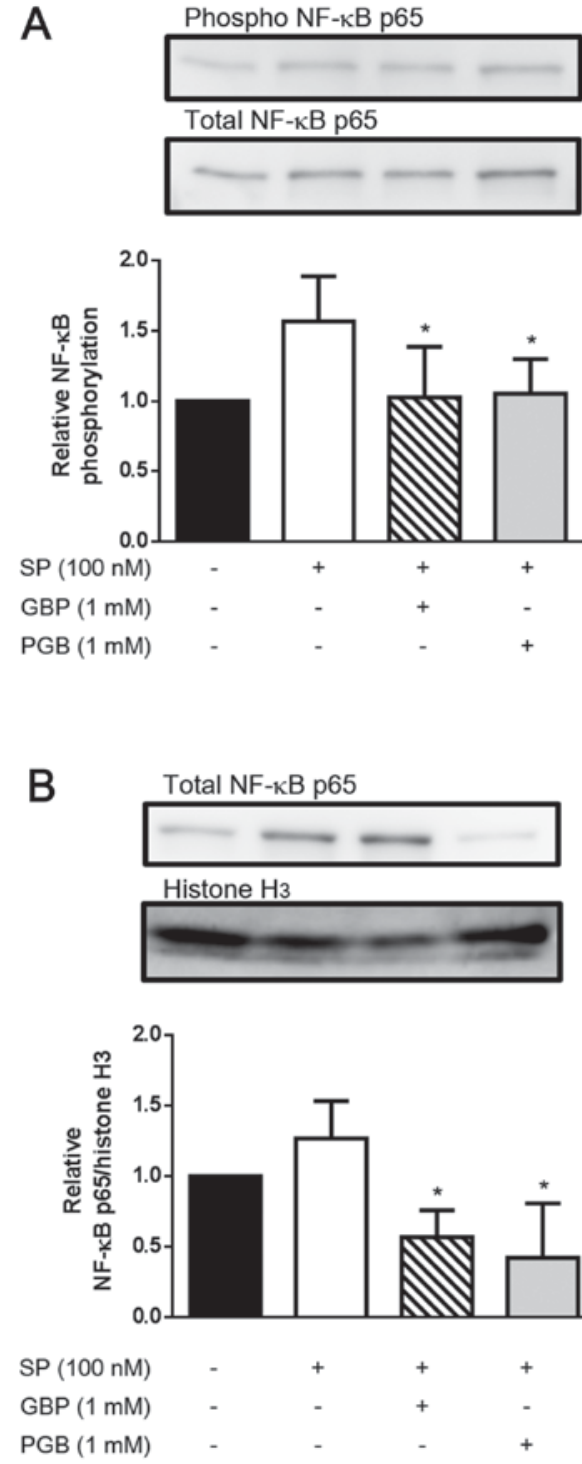

Figure 2. Evaluation of the effects of gabapentin (GBP) and pregabalin (PGB) on substance $P(\mathrm{SP})$-induced phosporylation of nuclear factor (NF) $\kappa \mathrm{B}$ and SP-induced nuclear translocation of NF- $\mathrm{NB}$. U373 MG cells were plated into 12 -well tissue culture plates at a density of $2 \times 10^{5}$ cells/well and incubated in minimum essential medium (MEM) with $10 \%$ FBS for $12 \mathrm{~h}$, followed by incubation in MEM with $0.5 \%$ fetal bovine serum (FBS) for $12 \mathrm{~h}$ at $37^{\circ} \mathrm{C}$. Subsequently, the cells were incubated with or without GBP $(1 \mathrm{mM})$ or PGB $(1 \mathrm{mM})$ for $60 \mathrm{~min}$, and then incubated with or without $\mathrm{SP}(100 \mathrm{nM})$ for $15 \mathrm{~min}$. (A) The expression of phosphorylation of NF- $\mathrm{B}$ were detected by probing with anti-phospho NF- $\mathrm{kB}$ p65 (Ser536) rabbit mAb and HRP-conjugated goat anti-rabbit immunoglobulin $\mathrm{G}(\mathrm{IgG})$. In order to confirm that equal amount of proteins were analyzed in each samples, the blots were stripped, and total $\mathrm{NF}-\kappa \mathrm{B}$ were detected by reprobing with anti-NF- $\mathrm{B}$ p 65 rabbit $\mathrm{mAb}$ and horseradish peroxidase (HRP)-conjugated goat anti-rabbit IgG. (B) After incubation of U373 MG cells with SP in the absence or presence of GBP or PGB, nuclear fractions were prepared, and the

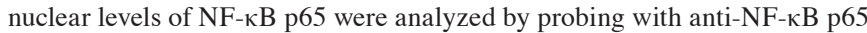
rabbit $\mathrm{mAb}$ and $\mathrm{HRP}$-conjugated goat anti-rabbit IgG. In order to confirm that equal amount of proteins were analyzed in each samples, the blots were stripped, and histone $\mathrm{H} 3$ were detected by reprobing with anti-histone $\mathrm{H} 3$ and HRP-conjugated goat anti-rabbit IgG. A representative image is shown. Data are the means \pm standard deviation of 3 separate experiments, and expressed as relative to the cells incubated without SP, GBP, and PGB. Data are compared between the SP-stimulated cells incubated without and with GBP or PGB. * $\mathrm{P}<0.05$.

epileptic seizures resistant to conventional therapies (12). However, subsequent single center and multicenter, randomized 

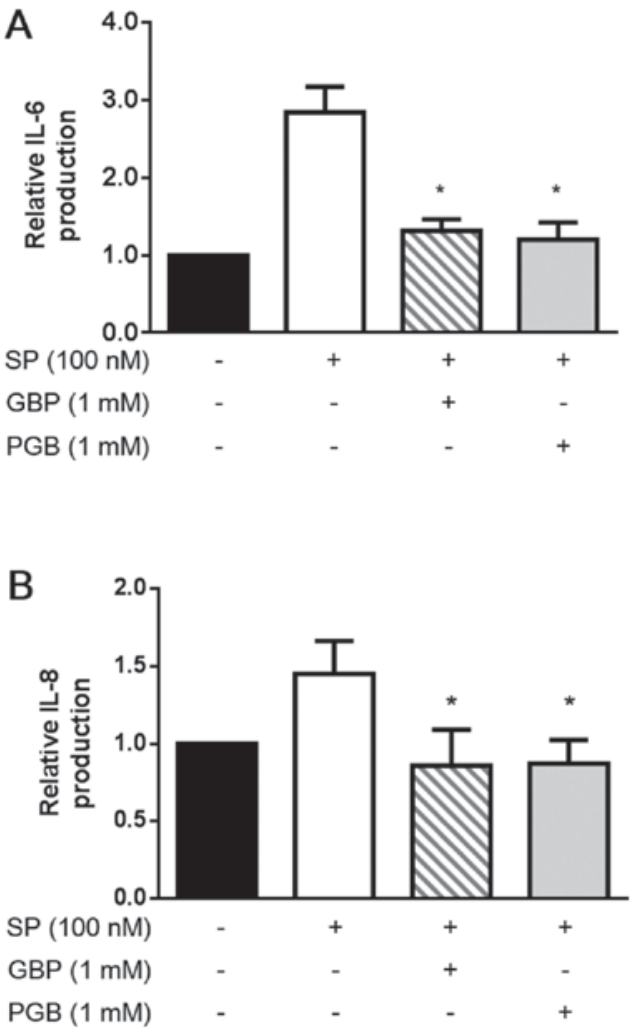

Figure 3. Evaluation of the effects of gabapentin (GBP) and pregabalin (PGB) on substance P (SP)-induced production of IL-6 and IL-8. U373 MG cells were plated into 12 -well tissue culture plates at a density of $1 \times 10^{5}$ cells/well and incubated in minimum essential medium (MEM) with $10 \%$ fetal bovine serum (FBS) for $12 \mathrm{~h}$, followed by incubation in MEM with $0.5 \%$ FBS for $12 \mathrm{~h}$ at $37^{\circ} \mathrm{C}$. Subsequently, the cells were incubated with or without GBP $(1 \mathrm{mM})$ or PGB $(1 \mathrm{mM})$ for $60 \mathrm{~min}$, and then incubated with or without SP $(100 \mathrm{nM})$. After $24 \mathrm{~h}$, culture supernatants were harvested, and the levels of (A) interleukin (IL)-6 and (B) IL-8 were measured by ELISA. Data are the means \pm standard deviation of 3 separate experiments, and expressed as relative to the cells incubated without SP, GBP, and PGB. Data are compared between the SP-stimulated cells incubated without and with GBP or PGB ${ }^{*} \mathrm{P}<0.05$.

double-blind trials demonstrated that GBP is also effective for the management of pain of inflammatory and neuropathic origin, such as post herpetic neuralgia and painful diabetic neuropathy $(39,40)$. Although PGB has not been extensively investigated as GBP, recent double-blind trials showed that PGB is also effective in the management of postoperative pain and diabetic neuropathy (39). In animal models of nociception, GBP reduces the mechanical or thermal hypersensitivity associated with nerve injury $(31,41)$, incisional injury (26), inflammatory injury $(27,41,42)$, and formalin-induced injury (42-44). PGB similarly reduces the mechanical or thermal hypersensitivity associated with injuries, described above $(27,42,45)$. In contrast, both compounds do not exhibit substantive effect on acute pain in uninjured animal models.

An interesting action of SP is the induction and modulation of proinflammatory cytokine secretion by glial cells (7). The mechanism of SP-induced signal transduction is incompletely understood. However, it has been suggested that the activation of NF- $\kappa \mathrm{B}$ is involved in SP-induced cytokine expression (7). Importantly, it is demonstrated that GBP and PGB decrease SP-induced NF- $\kappa \mathrm{B}$ activation in human neuroblastoma and rat glioma cells, and that these drugs also inhibit NF- $\kappa \mathrm{B}$ activation in rat spinal dorsal root ganglia cells pre-treated with SP (38). Interestingly, it has been reported that GBP and PGB attenuate the release of neuropeptides (such as SP) from inflamed spinal cord (34). Importantly, the present study demonstrated that GBP and PGB prevent the SP-induced IL- 6 and IL- 8 production in U373 MG cells by inhibiting p38 MAPK and NF- $\kappa \mathrm{B}$ activation. Based on these findings, it could be speculated that gabapentinoids may exhibit anti-inflammatory action by suppressing not only the SP-induced cytokine production via the inhibition of p38 MAPK and NF- $\kappa \mathrm{B}$ activation, but also the secretion of inflammatory neuropeptides (such as SP) at the inflamed neural tissue. In fact, it has been demonstrated that PGB binds potently to the $\alpha 2 \delta-1$ subunit of calcium channels and modulates calcium influx at nerve terminals, thereby reducing the release of several neurotransmitters, including SP (46). However, it has been also reported that PGB and GBP suppress the degradation of $\mathrm{I} \kappa \mathrm{B}$, thereby inhibiting nuclear localization of NF- $\kappa \mathrm{B}$ (p65) in SH-SY5Y glioblastoma cells (45). Thus, gabapentinoid-mediated modulation of nuclear localization of NF- $\mathrm{BB}$ is also considered as a molecular mechanism for the actions of GBP and PGB.

The $\alpha 2 \delta-1$ subunit of VGCC is involved in propagation of excitatory signals mediated by glutamate, calcitonin gene-related protein (CGRP), and SP (34). Its upregulation under pathological conditions is associated with hyperexcitatory states such as seizures and neuropathic pain. The causal link between $\alpha 2 \delta$-1-subunit expression and hyperalgesia has been demonstrated in several experimental animal models of spinal nerve injury $(47,48)$. Notably, PGB binds potently to the $\alpha 2 \delta-1$ subunit and suppresses calcium influx at nerve terminals, thereby modulating hyperexcitatory states of neuronal synapse.

SP stimulates a number of intracellular signaling molecules including the members of MAPK family (ERK1/2 and p38 MAPK) via the action of NK1R. Moreover, SP induce the IL-6 and IL-8 production via the activation of p38 MAPK, ERK1/2 and NF- $\kappa$ B by U373 MG (49). In this study, GBP and PGB did not reduce the SP-induced ERK1/2 phosphorylation (Fig. 1B) but reduced p38 MAPK phosphorylation in U373 MG cells (Fig. 1A). Thus, our results suggest that GBP and PGB reduce the SP-induced production of IL- 6 and IL-8 by the NK1R (a principal receptor for SP)-expressing U373 MG cells via the inhibition of signaling molecules p38 MAPK but not ERK1/2. It is possible that GBP and PGB may reduce the expression of NK1R, thereby suppressing the SP-induced production of IL-6 and IL-8. However, GBP and PGB did not reduce the SP-induced ERK1/2 phosphorylation, as mentioned above. Thus, GBP and PGB unlikely modulate the expression of NK1R.

In a neuropathic pain model, it is well documented that microglial activation, accompanying with p38 MAPK phosphorylation in the spinal cord, plays an important role in the development of neuropathic pain (50-52). Both peripheral inflammation and nerve injury induce p38 MAPK activation in spinal microglia, and p38 MAPK inhibitor SB203580 suppresses the inflammation-induced thermal hyperalgesia and spinal nerve ligation-induced mechanical allodynia (53). Moreover, intrathecal injection of minocycline, which inhibits spinal microglia activation by blocking p38 MAPK, exerts antinociceptive effect in both inflammation and neuropathic 
pain models (52). These observations indicate that p38 MAPK activation plays important role in development of neuroinflammation. In addition, IL- 6 is speculated to be involved in the development of neuropathic pain, because IL-6 mRNA was significantly elevated in both the dorsal and ventral horns in a neuropathic pain model of spinal nerve cryoneurolysis and spinal nerve tight ligation $(54,55)$. IL- 8 also contributes to the pathophysiology of inflammatory pain, because the expression of IL-8 was critically upregulated in the ipsilateral spinal cord dorsal horn after chronic constriction injury $(56,57)$. In this study, GBP and PGB reduced IL- 6 and IL- 8 production possibly by suppressing p38 MAPK in U373 MG cell, and the results explain the pharmacological action of GBP and PGB on neuroinflammation (neuropathic pain).

In conclusion, we demonstrated that GBP and PGB suppressed the production of IL-6 and IL-8 in U373 MG cells. Furthermore, GBP and PGB inhibited the SP-induced activation of $\mathrm{p} 38 \mathrm{MAPK}$, and NF- $\mathrm{NB}$ in U373 MG cells. Thus, GBP and PGB likely prevent the SP-induced IL- 6 and IL-8 production by U373 MG cells via the inhibition of signaling molecules including p38 MAPK and NF- $\kappa$ B, thereby exhibiting anti-neuroinflammatory action.

\section{Acknowledgements}

This study was supported by Grant-in-Aid for Scientific Research (C) (15K10564).

\section{References}

1. Samsam M, Coveñas R, Ahangari R, Yajeya J, Narváez JA and Tramu G: Simultaneous depletion of neurokinin A, substance P and calcitonin gene-related peptide from the caudal trigeminal nucleus of the rat during electrical stimulation of the trigeminal ganglion. Pain 84: 389-395, 2000.

2. Johnson MB, Young AD and Marriott I: The therapeutic potential of targeting substance P/NK-1R interactions in inflammatory CNS disorders. Front Cell Neurosci 10: 296, 2017.

3. Muñoz M and Coveñas R: Involvement of substance $P$ and the NK-1 receptor in cancer progression. Peptides 48: 1-9, 2013.

4. Ebner K and Singewald N: The role of substance P in stress and anxiety responses. Amino Acids 31: 251-272, 2006.

5. Garcia-Recio S and Gascón P: Biological and pharmacological aspects of the NK1-receptor. Biomed Res Int 2015: 495704, 2015.

6. Lieb K, Schaller H, Bauer J, Berger M, Schulze-Osthoff K and Fiebich BL: Substance P and histamine induce interleukin-6 expression in human astrocytoma cells by a mechanism involving protein kinase $\mathrm{C}$ and nuclear factor-IL-6. J Neurochem 70: $1577-1583,1998$

7. Lieb K, Fiebich BL, Berger M, Bauer J and Schulze-Osthoff K: The neuropeptide substance $\mathrm{P}$ activates transcription factor NF-kappa B and kappa B-dependent gene expression in human astrocytoma cells. J Immunol 159: 4952-4958, 1997.

8. Horuk R, Martin AW, Wang Z, Schweitzer L, Gerassimides A, Guo H, Lu Z, Hesselgesser J, Perez HD, Kim J, et al: Expression of chemokine receptors by subsets of neurons in the central nervous system. J Immunol 158: 2882-2890, 1997.

9. Kim SY, Bae JC, Kim JY, Lee HL, Lee KM, Kim DS and Cho HJ: Activation of p38 MAP kinase in the rat dorsal root ganglia and spinal cord following peripheral inflammation and nerve injury. Neuroreport 13: 2483-2486, 2002.

10. Ji RR, Befort K, Brenner GJ and Woolf CJ: ERK MAP kinase activation in superficial spinal cord neurons induces prodynorphin and NK-1 upregulation and contributes to persistent inflammatory pain hypersensitivity. J Neurosci 22: 478-585, 2002.

11. Jin SX, Zhuang ZY, Woolf CJ and Ji RR: p38 mitogen-activated protein kinase is activated after a spinal nerve ligation in spinal cord microglia and dorsal root ganglion neurons and contributes to the generation of neuropathic pain. J Neurosci 23: 4017-4022, 2003 .
12. Bryans JS and Wustrow DJ: 3-substituted GABA analogs with central nervous system activity: a review. Med Res Rev 19: 149-177, 1999.

13. Taylor CP, Gee NS, Su TZ, Kocsis JD, Welty DF, Brown JP, Dooley DJ, Boden P and Singh L: A summary of mechanistic hypotheses of gabapentin pharmacology. Epilepsy Res 29: 233-249, 1998

14. Maneuf YP, Gonzalez MI, Sutton KS, Chung FZ, Pinnock RD and Lee K: Cellular and molecular action of the putative GABA-mimetic, gabapentin. Cell Mol Life Sci 60: 742-750, 2003.

15. Brown JT and Randall A: Gabapentin fails to alter P/Q-type $\mathrm{Ca}^{2+}$ channel-mediated synaptic transmission in the hippocampus in vitro. Synapse 55: 262-269, 2005.

16. SILLS G: The mechanisms of action of gabapentin and pregabalin. Curr Opin Pharmacol 6: 108-113, 2006.

17. Maneuf YP, Hughes J and McKnight AT: Gabapentin inhibits the substance P-facilitated K (+)-evoked release of [(3)H]glutamate from rat caudial trigeminal nucleus slices. Pain 93: 191-196, 2001.

18. Cunningham MO, Woodhall GL, Thompson SE, Dooley DJ and Jones RSG: Dual effects of gabapentin and pregabalin on glutamate release at rat entorhinal synapses in vitro. Eur J Neurosci 20: 1566-1576, 2004.

19. Dooley DJ, Mieske CA and Borosky SA: Inhibition of $\mathrm{K}(+)$-evoked glutamate release from rat neocortical and hippocampal slices by gabapentin. Neurosci Lett 280: 107-110, 2000.

20. Rosenberg JM, Harrell C, Ristic H, Werner RA and de Rosayro AM: The effect of gabapentin on neuropathic pain. Clin J Pain 13: 251-255, 1997.

21. Backonja M, Beydoun A, Edwards KR, Schwartz SL, Fonseca V, Hes M, LaMoreaux L and Garofalo E: Gabapentin for the symptomatic treatment of painful neuropathy in patients with diabetes mellitus: A randomized controlled trial. JAMA 280: 1831-1836, 1998.

22. Rowbotham M, Harden N, Stacey B, Bernstein P and Magnus-Miller L: Gabapentin for the treatment of postherpetic neuralgia: A randomized controlled trial. JAMA 280: 1837-1842, 1998.

23. Dirks J, Fredensborg BB, Christensen D, Fomsgaard JS, Flyger H and Dahl JB: A randomized study of the effects of single-dose gabapentin versus placebo on postoperative pain and morphine consumption after mastectomy. Anesthesiology 97: 560-564, 2002.

24. Serpell MG; Neuropathic pain study group: Gabapentin in neuropathic pain syndromes: A randomised, double-blind, placebo-controlled trial. Pain 99: 557-566, 2002.

25. Werner MU, Perkins FM, Holte K, Pedersen JL and Kehlet H: Effects of gabapentin in acute inflammatory pain in humans. Reg Anesth Pain Med 26: 322-328.

26. Field MJ, Holloman EF, McCleary S, Hughes J and Singh L: Evaluation of gabapentin and S-(+)-3-isobutylgaba in a rat model of postoperative pain. J Pharmacol Exp Ther 282: 1242-1246, 1997.

27. Houghton AK, Lu Y and Westlund KN: S-(+)-3-isobutylgaba and its stereoisomer reduces the amount of inflammation and hyperalgesia in an acute arthritis model in the rat. J Pharmacol Exp Ther 285: 533-538, 1998.

28. Partridge BJ, Chaplan SR, Sakamoto E and Yaksh TL: Characterization of the effects of gabapentin and 3-isobutyl-gamma-aminobutyric acid on substance P-induced thermal hyperalgesia. Anesthesiology 88: 196-205, 1998.

29. Chen SR, Xu Z and Pan HL: Stereospecific effect of pregabalin on ectopic afferent discharges and neuropathic pain induced by sciatic nerve ligation in rats. Anesthesiology 95: 1473-1479, 2001.

30. Takasaki I, Andoh T, Nojima H, Shiraki K and Kuraishi Y: Gabapentin antinociception in mice with acute herpetic pain induced by herpes simplex virus infection. J Pharmacol Exp Ther 296: 270-275, 2001.

31. Hunter JC, Gogas KR, Hedley LR, Jacobson LO, Kassotakis L, Thompson J and Fontana DJ: The effect of novel anti-epileptic drugs in rat experimental models of acute and chronic pain. Eur J Pharmacol 324: 153-160, 1997.

32. Stanfa LC, Singh L, Williams RG and Dickenson AH: Gabapentin, ineffective in normal rats, markedly reduces C-fibre evoked responses after inflammation. Neuroreport 8: 587-590, 1997.

33. Oku R, Satoh M and Takagi H: Release of substance $\mathrm{P}$ from the spinal dorsal horn is enhanced in polyarthritic rats. Neurosci Lett 74: 315-319, 1987. 
34. Fehrenbacher JC, Taylor CP and Vasko MR: Pregabalin and gabapentin reduce release of substance P and CGRP from rat spinal tissues only after inflammation or activation of protein kinase C. Pain 105: 133-141, 2003.

35. Heuillet E, Ménager J, Fardin V, Flamand O, Bock M, Garret C, Crespo A, Fallourd AM and Doble A: Characterization of a human NK1 tachykinin receptor in the astrocytoma cell line U 373 MG. J Neurochem 60: 868-876, 1993.

36. Bahrami G and Mohammadi B: Sensitive microanalysis of gabapentin by high-performance liquid chromatography in human serum using pre-column derivatization with 4-chloro-7-nitrobenzofurazan: Application to a bioequivalence study. J Chromatogr B Analyt Technol Biomed Life Sci 837: 24-28, 2006.

37. Jalalizadeh H, Souri E, Tehrani MB and Jahangiri A: Validated HPLC method for the determination of gabapentin in human plasma using pre-column derivatization with 1-fluoro-2,4-dinitrobenzene and its application to a pharmacokinetic study. J Chromatogr B Analyt Technol Biomed Life Sci 854: 43-47, 2007.

38. Park S, Ahn ES, Han DW, Lee JH, Min KT, Kim H and Hong YW: Pregabalin and gabapentin inhibit substance P-induced NF-kappaB activation in neuroblastoma and glioma cells. J Cell Biochem 105: 414-423, 2008.

39. Tremont-Lukats IW, Megeff $\mathrm{C}$ and Backonja $\mathrm{MM}$ Anticonvulsants for neuropathic pain syndromes: Mechanisms of action and place in therapy. Drugs 60: 1029-1052, 2000.

40. Mao J and Chen LL: Gabapentin in pain management. Anesth Analg 91: 680-687, 2000.

41. Patel S, Naeem S, Kesingland A, Froestl W, Capogna M, Urban L and Fox A: The effects of GABA(B) agonists and gabapentin on mechanical hyperalgesia in models of neuropathic and inflammatory pain in the rat. Pain 90: 217-226, 2001.

42. Field MJ, Oles RJ, Lewis AS, McCleary S, Hughes J and Singh L: Gabapentin (neurontin) and S-(+)-3-isobutylgaba represent a novel class of selective antihyperalgesic agents. $\mathrm{Br} \mathbf{J}$ Pharmacol 121: 1513-1522, 1997.

43. Shimoyama N, Shimoyama M, Davis AM, Inturrisi CE and Elliott KJ: Spinal gabapentin is antinociceptive in the rat formalin test. Neurosci Lett 222: 65-67, 1997.

44. Kaneko M, Mestre C, Sánchez EH and Hammond DL: Intrathecally administered gabapentin inhibits formalin-evoked nociception and the expression of Fos-like immunoreactivity in the spinal cord of the rat. J Pharmacol Exp Ther 292: 743-751, 2000 .
45. Field MJ, McCleary S, Hughes J and Singh L: Gabapentin and pregabalin, but not morphine and amitriptyline, block both static and dynamic components of mechanical allodynia induced by streptozocin in the rat. Pain 80: 391-398, 1999.

46. Gajraj NM: Pregabalin: Its pharmacology and use in pain management. Anesth Analg 105: 1805-1815,2007.

47. Davies A, Hendrich J, Van Minh AT, Wratten J, Douglas L and Dolphin AC: Functional biology of the alpha(2)delta subunits of voltage-gated calcium channels. Trends Pharmacol Sci 28: 220-228, 2007.

48. Luo ZD, Chaplan SR, Higuera ES, Sorkin LS, Stauderman KA, Williams ME and Yaksh TL: Upregulation of dorsal root ganglion (alpha)2(delta) calcium channel subunit and its correlation with allodynia in spinal nerve-injured rats. J Neurosci 21: 1868-1875, 2001

49. Fiebich BL, Schleicher S, Butcher RD, Craig A and Lieb K: The neuropeptide substance $\mathrm{P}$ activates p38 mitogen-activated protein kinase resulting in IL-6 expression independently from NF-kappa B. J Immunol 165: 5606-5611, 2000.

50. Hu P, Bembrick AL, Keay KA and McLachlan EM: Immune cell involvement in dorsal root ganglia and spinal cord after chronic constriction or transection of the rat sciatic nerve. Brain Behav Immun 21: 599-616, 2007.

51. Scholz J, Abele A, Marian C, Häussler A, Herbert TA, Woolf CJ and Tegeder I: Low-dose methotrexate reduces peripheral nerve injury-evoked spinal microglial activation and neuropathic pain behavior in rats. Pain 138: 130-142, 2008.

52. Cao H and Zhang YQ: Spinal glial activation contributes to pathological pain states. Neurosci Biobehav Rev 32: 972-983, 2008.

53. Obata K and Noguchi K: MAPK activation in nociceptive neurons and pain hypersensitivity. Life Sci 74: 2643-2653, 2004.

54. Arruda JL, Colburn RW, Rickman AJ, Rutkowski MD and DeLeo JA: Increase of interleukin- 6 mRNA in the spinal cord following peripheral nerve injury in the rat: Potential role of IL-6 in neuropathic pain. Brain Res Mol Brain Res 62: 228-235, 1998

55. Lee HL, Lee KM, Son SJ, Hwang SH and Cho HJ: Temporal expression of cytokines and their receptors mRNAs in a neuropathic pain model. Neuroreport 15: 2807-2811, 2004.

56. Wang XM, Hamza M, Wu TX and Dionne RA: Upregulation of IL-6, IL-8 and CCL2 gene expression after acute inflammation: Correlation to clinical pain. Pain 142: 275-283, 2009.

57. Wang F, Xu S, Shen X, Guo X, Peng Y and Yang J: Spinal macrophage migration inhibitory factor is a major contributor to rodent neuropathic pain-like hypersensitivity. Anesthesiology 114: 643-659, 2011 . 